\title{
Magnetic resonance imaging of the behaviour of oil-in-water emulsions in the gastric lumen of man
}

\author{
Luca Marciani ${ }^{1,2}$, Martin S. J. Wickham ${ }^{3}$, Debbie Bush ${ }^{4}$, Richard Faulks ${ }^{3}$, Jeff Wright ${ }^{4}$, \\ Annette J. Fillery-Travis ${ }^{3,5}$, Robin C. Spiller ${ }^{1}$ and Penny A. Gowland ${ }^{2 *}$ \\ ${ }^{1}$ Wolfson Digestive Diseases Centre, Queen's Medical Centre, University Hospital, Nottingham NG7 2UH, UK \\ ${ }^{2}$ Sir Peter Mansfield Magnetic Resonance Centre, School of Physics and Astronomy, University of Nottingham, Nottingham NG7 \\ $2 R D, U K$ \\ ${ }^{3}$ Institute of Food Research, Colney, Norwich NR4 7UA, UK \\ ${ }^{4}$ Department of Surgery, Queen's Medical Centre, University Hospital, Nottingham NG7 2UH, UK \\ ${ }^{5}$ Centre for Research and Dissemination, PDF.net, 39 Harvey Lane, Norwich NR7 OBZ, UK
}

(Received 9 May 2005 - Revised 12 September 2005 - Accepted 18 September 2005)

\begin{abstract}
Pre-processed foods often contain a high percentage of lipid, present as emulsions stabilised with various surface-active agents. The acidic gastric environment can affect the behaviour of such emulsions, modifying the lipid spatial distribution and, in turn, the rate of gastric emptying and nutrient delivery to the gut. The aim of the present study was to use echo-planar magnetic resonance imaging (EPI) to determine the behaviour of model olive oil emulsions during gastric processing. Six healthy male volunteers were intubated nasogastrically on two separate occasions and fed $500 \mathrm{ml} 15 \%(\mathrm{w} / \mathrm{w})$ olive oil-in-water, surfactant-stabilised emulsions designed to have identical droplet size distribution and which were either stable or unstable under gastric acid conditions. EPI was used to assess the oil fraction of the intragastric emulsions, gastric emptying and to visualise the spatial distribution of the oil at 10,30 and $50 \mathrm{~min}$ postprandially. The in vivo imaging measurements of the oil volume fraction of the emulsions correlated well ( $r$ 0.66, acid-stable; $r 0.52$, acid-unstable) with that assayed in the gastric aspirates. Compared with the acid-stable emulsion, the acid-unstable emulsion in the gastric lumen rapidly separated into lipid-depleted 'aqueous' and lipid layers. Phase separation in the acidunstable meal allowed the oil-depleted component to empty first and more rapidly than the stable emulsion as determined by the gastric emptying curves. These pilot data suggest that gastric processing and emptying of high-fat foods could be manipulated by careful choice of emulsifier.
\end{abstract}

Magnetic resonance imaging: Echo-planar imaging: Stomach: Man

Foods with a high fat content are characterised by high energy density and palatability (Drewnowski, 1997) and cross-sectional studies have shown that relative body mass is positively associated with the concentration of fat within the diet (Lissner \& Heitmann, 1995). The digestion of fat is, however, a complex, energy-dependent process that requires precise regulation to prevent overwhelming the absorptive capacity of the small intestine. Thus gastric emptying and delivery of fat to the small intestine are precisely controlled by a negative feedback from NEFA in the duodenum. Delivery of NEFA to the duodenum depends on posture (Horowitz et al. 1993) and on lipolysis, so that pancreatic insufficiency (Carney et al. 1995) and lipase inhibition accelerate gastric emptying (Borovicka et al. 2000; Pilichiewicz et al. 2003). The intestinal feedback also depends on the length of the small intestine exposed (Lin et al. 1990). NEFA in the duodenum release cholecystokinin, which stimulates pancreatic and biliary secretions acting via enteric nerves and inhibits gastric emptying via the vagus.
Previous studies have investigated the emptying of different food components, either solid (Meyer et al. 1976; Malmud et al. 1982; Christian et al. 1984; Collins et al. 1988; Kerlin et al. 1989) or liquid (Heading et al. 1971; Malmud et al. 1982) from the stomach. Fewer data are available on the gastric handling of fat, although by use of radio-pharmaceuticals designed to label the fat component of a meal (Jian et al. 1982; Cunningham et al. 1991) it has been possible to assess gastric emptying of fat with $\gamma$-scintigraphy (Horowitz et al. 1985). Such studies have provided evidence that the isolated lipid component of a test meal empties much more slowly than the aqueous component of the meal (Jian et al. 1982; Cunningham et al. 1991), whilst lipid integrated within the matrix of solid food particles empties with the solid phase of a meal (Edelbroek et al. 1992). However, these studies have not generally used surfactant-stabilised oil-in-water emulsions, which are commonly used by the food industry. The National Food Survey 2000 (DEFRA, 2000) indicated that fat consumption in the UK is around $75 \mathrm{~g}$ per $\mathrm{d}$ per person, the majority of which is ingested

Abbreviations: EPI, echo-planar imaging; MRI, magnetic resonance imaging; $T_{1}$, longitudinal relaxation time; $T_{2}$, transverse relaxation time; $t_{50} \%$, time to half empty the gastric contents.

* Corresponding author: Professor Penny A. Gowland, fax: +44 115951 5166, email Penny.Gowland@nottingham.ac.uk 
as natural emulsions (milk, cream) or as emulsions created during domestic or commercial food processing (for example, low- and reduced-fat spreads, sauces, dressings, etc.). Free added fat can also be present as carryover from food preparation, e.g. frying, but emulsification during mastication and gastric processing is part of the normal digestive process. In the light of the rising trend to obesity in the developed world (Mokdad et al. 2003), fuelled in part by the availability and palatability of high-fat foods, it is important to understand the intragastric behaviour of emulsion systems (Dickinson, 2003).

Recently, magnetic resonance imaging (MRI; Schwizer et al. 1992; Fraser et al. 1993; Kunz et al. 2005) and in particular its ultra-fast variant echo-planar imaging (EPI; Howseman et al. 1988) have been used successfully to investigate non-invasively gastric emptying and motility (Stehling et al. 1989; Evans et al. 1993; Marciani et al. 2001a) and effects of intragastric fat on antral flow and emptying (Boulby et al. 1997, 1999). EPI is non-invasive, provides images with high resolution and good contrast between different food components, and does not need to use radioactive or pharmacological contrast agents. It would therefore be advantageous to be able to use EPI techniques to assess the intragastric distribution and oil volume fraction of oil emulsions in vivo, serially and non-invasively, and to relate these properties to gastrointestinal function and postprandial plasma lipid, gut signalling peptides response and, ultimately, to satiety. We have recently developed a method to measure the oil fraction in oil-in-water emulsions in vivo within the gastric lumen using inversion recovery EPI and a two non-exchanging compartments empirical model (Marciani et al. 2004).

The aims of the present study were to develop two oilin-water emulsion test meals that differed only in their stability within the acidic gastric environment; to apply noninvasive, ultra-fast MRI techniques to monitor their intragastric behaviour and gastric emptying; and to validate the in vivo oil fraction measurements by MRI against gastric aspirates for both emulsion meals (Marciani et al. 2004).

\section{Materials and methods}

\section{Meal preparation}

The present study required the production of two oil-in-water emulsion systems with equal lipid content (and therefore energy density) and equal lipid droplet size distributions, but contrasting acid stabilities. One emulsion meal was required to stay intact when exposed to the acidic conditions experienced during gastric processing, retaining both its emulsion structure and distribution of oil droplet dimensions. The second emulsion meal was required to destabilise when exposed to the low gastric $\mathrm{pH}$, breaking into two distinct phases (a lipid phase and an aqueous phase). These contrasting acid stabilities were achieved by using two closely related, commonly used food-grade emulsifiers: polyoxyethylene sorbitan monostearate (Tween 60, E491; Macphie, Glenbervie, UK) for the acid-stable emulsion and sorbitan monooleate (Span 80, E494; Esterchem Ltd, Leek, UK) for the acidunstable emulsion. These two emulsifiers have a very similar chemical structure (Fig. 1) and differ only in a polyoxyethylene group and a double bond on the fatty acid chain.
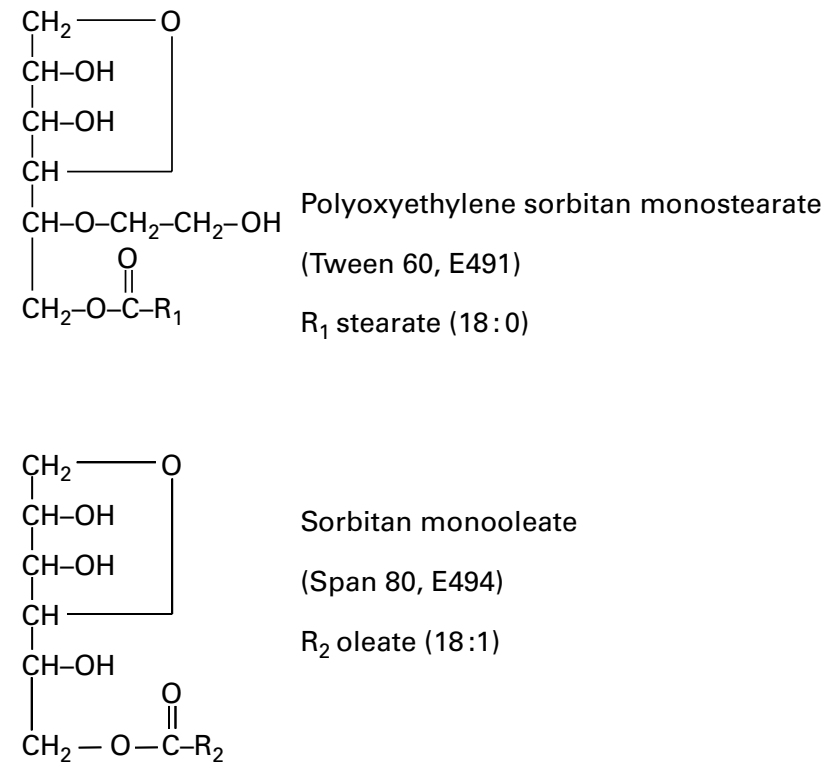

Sorbitan monooleate

(Span 80, E494)

$\mathrm{R}_{2}$ oleate $(18: 1)$

Fig. 1. Chemical structure of the Tween 60 and Span 80 emulsifiers. The two emulsifiers used in the present study have very similar chemical structure and differ only in a polyoxyethylene group and a double bond on the fatty acid chain. For details of subjects and procedures, see this page.

The emulsion meals were prepared at room temperature by mixing $12.5 \mathrm{~g}$ food-grade Tween 60 or $12.5 \mathrm{~g}$ food-grade Span 80 , trace amounts of non-energetic sweetener (Hermesetas Gold; McCormick Foods Australia Ltd, Clayton South, Australia) and coffee flavouring (Supercook Flavouring, Leeds, $\mathrm{UK}$ ) and $75 \mathrm{~g}$ food-grade extra-virgin olive oil, and made up to $500 \mathrm{ml}$ with sterile deionised water. They were then blended at full speed in a Waring PB20E blender (Waring, Torrington, CT, USA) for $90 \mathrm{~s}$. The emulsion meals were allowed to equilibrate with gentle stirring at $37^{\circ} \mathrm{C}$ for $15 \mathrm{~min}$ in a water bath. The energy content of the meals, taking into account the presence of the emulsifiers, was $3150 \mathrm{~kJ}$ (of which $2835 \mathrm{~kJ}$ was from the olive oil). The Tween 60 has an additional oxyethylene ester that may have provided an additional $12 \mathrm{~kJ}$.

\section{In vitro validation of meal behaviour}

For the in vitro validation of the behaviour of the emulsions under acidic conditions, both an acid-stable and an acidunstable meal were prepared as described earlier. A $75 \mathrm{~g}$ sample of each meal was placed into a beaker and held at $37^{\circ} \mathrm{C}$ and slowly but continuously stirred with magnetic stirrer bars. The $\mathrm{pH}$ of the emulsion meals was continuously monitored using $\mathrm{pH}$ electrodes. The emulsions were slowly acidified from their native $\mathrm{pH}$ (approximately 5.30) to a final $\mathrm{pH}$ of 2.0 by drop-wise addition of $1-\mathrm{M} \mathrm{HCl}$. The rate of change of $\mathrm{pH}$ against volume of acid added was the same for both emulsions. The Span 80-stabilised emulsion rapidly broke into two distinct phases (oil on top of the water) as the $\mathrm{pH}$ was reduced to below 4.0. Once acidified to $\mathrm{pH} 2 \cdot 0$, a sample was collected from each of the two meals (for the Span 80-stabilised meal, the aqueous phase was sampled) and the mean oil droplet size distributions were measured. Subsequently, to assess the temporal stability of both emulsion systems, they were left under stirring at $\mathrm{pH} 2.0$ and $37^{\circ} \mathrm{C}$ for a 
further $60 \mathrm{~min}$. A further sample was collected from each of the meals (again for the Span 80-stabilised meal, the aqueous phase was sampled) and the mean oil droplet size distributions were again measured.

\section{Measurement of lipid droplet size of meals}

The dispersed-phase droplet size distribution of both emulsions was determined using a Coulter LS230 (Beckman Coulter, High Wycombe, Buckinghamshire, UK). This instrument can measure particles in solution with diameters between 0.004 and $2000 \mu \mathrm{m}$ using enhanced laser diffraction (excitation $750 \mathrm{~nm}$ ) and polarisation intensity differential scattering. An aliquot of an emulsion meal was diluted with sterile deionised water. The emulsion attenuates the laser light passing through the sample. Therefore, the dilution of the emulsion meal required for each measurement was selected to produce attenuation between 8 and $16 \%$. Light scattering data were then collected for $60 \mathrm{~s}$. The data were then transformed to a lipid droplet size distribution using an optical model that is consistent with the refractive index of olive oil (1.456). Each measurement was performed in triplicate with fresh aliquots of the test emulsion meals.

\section{Zeta potential of meal emulsion oil droplets}

The electric potential at the oil droplets' surface of shear (zeta potential) was measured using a Malvern Instruments ZetaSizer (Malvern Instruments, Malvern, UK). The zeta potential is calculated by the Henry formulation from the mobility of a droplet within an electric field (Shaw, 1992). Samples of the acid-stable and acid-unstable meals after production, and of the acid-stable meal after $1 \mathrm{~h}$ at $\mathrm{pH} 2 \cdot 0$, were used for this measurement. The technique required a low volume fraction for the emulsion $(0.03 \% \mathrm{w} / \mathrm{w})$; hence, the samples were diluted with sterile deionised water.

\section{Volunteers and study design}

Six healthy male volunteers were recruited. They were 25 (SE 3) years old with no history of gastrointestinal or metabolic disorders as determined by questionnaire and were within $\pm 25 \%$ of ideal body weight for their height (BMI 23 $(\mathrm{SE} 1) \mathrm{kg} / \mathrm{m}^{2}$ ). The volunteers attended on two separate experimental morning sessions (more than $1 \mathrm{~d}$ and less than $7 \mathrm{~d}$ apart), having fasted overnight $(>12 \mathrm{~h})$ on each occasion. They were intubated with a nasogastric tube (internal diameter $4 \mathrm{~mm}$ ), which was positioned so that its tip lay on the greater curvature of the stomach with the subject in the left lateral position. Water $(50 \mathrm{ml})$ was then instilled and the positioning of the nasogastric tube within the gastric lumen was verified by recovering at least $45 \mathrm{ml}$ gastric aspirate, the acid $\mathrm{pH}$ of which was confirmed using indicator paper. This ensures that the nasogastric tube tip lies in the dependent portion of the stomach (midpoint of greater curvature or beyond). After this procedure, each volunteer drank one of the two emulsion meals $(500 \mathrm{ml})$, given in random order and which had been prepared as described earlier. The volunteers were asked to drink the emulsion meals within $10 \mathrm{~min}$. The time when meal ingestion started was defined as $t=0 \mathrm{~min}$. At $t=10$, 30 and $50 \mathrm{~min}$ the subjects underwent imaging and had a
$5 \mathrm{ml}$ gastric aspirate taken, with the first few $\mathrm{ml}$ aspirate withdrawn being discarded. The aspiration procedure took only about $20 \mathrm{~s}$ to perform. At $t=70 \mathrm{~min}$ the subjects drank $500 \mathrm{ml}$ water to dilute the emulsion remaining in the stomach and a final set of images and a final gastric aspirate were taken.

\section{Ethical considerations}

The test meal volume of $500 \mathrm{ml}$ containing $75 \mathrm{~g}$ olive oil was considered comparable to a large conventional meal. The insertion of a nasogastric tube and the serial aspiration of samples of the gastric contents are procedures that carry a very small risk of an adverse event and were performed by a nurse experienced in the procedure. MRI scanning is a non-invasive procedure and is well tolerated. This study was approved by the Nottingham Medical School Research Ethics Committee in accordance with the Declaration of Helsinki (2000). Volunteers gave informed written consent prior to experiments.

\section{Echo-planar imaging measurements}

Single-shot EPI (Howseman et al. 1988) images were acquired with the use of a whole-body $0.5 \mathrm{~T}$ purpose-built EPI scanner equipped with actively shielded gradients and a $50 \mathrm{~cm}$ diameter bird-cage coil. The in-plane resolution was $3.5 \mathrm{~mm} \times 2.5 \mathrm{~mm}$ and a slice thickness of $1 \mathrm{~cm}$ was used throughout the experiments. Each image was acquired in $130 \mathrm{~ms}$ using a $128 \times 128$ matrix with an effective echo time of $40 \mathrm{~ms}$. A transverse, rapid, multi-slice set of EPI images of the subject was acquired from the heart to the kidneys to determine the position and volume of the gastric lumen. The total acquisition time was only a few seconds. Longitudinal relaxation time $\left(T_{1}\right)$ measurements to measure oil fraction were carried out using single-shot inversion recovery EPI on a single slice, positioned across the body of the stomach. Although the nasogastric tube is seen generally well in the EPI volume sets, its tip is usually more difficult to detect. For the $T_{1}$ measurements we chose an imaging plane that provided the largest amount of meal in order to produce a more accurate assessment of $T_{1} . T_{1}$ is the time constant describing the recovery in signal intensity as a function of a specific time delay in the imaging sequence (called the inversion time). $T_{1}$ depends on the mobility of the protons being imaged and it differs between fat and water and also between different body tissues. A hyperbolic secant inversion pulse and eight inversion times varying from 23 to $2000 \mathrm{~ms}$ were used. The repetition time was $25 \mathrm{~s}$ with a total acquisition time of about $4.5 \mathrm{~min}$. On the same slice eight transverse relaxation time $\left(T_{2}\right)$-weighted spinecho EPI images (with eight echo times varying from 60 to $960 \mathrm{~ms}$ repeated once) were also acquired in order to observe creaming (a change in the spatial distribution of lipid due to the aggregation of oil droplets, which have a tendency to float to the surface unless gently mixed). $T_{2}$ is a time constant describing the decline in signal intensity on the images as a function of another specific time delay in the imaging sequence (called the echo time). $T_{2}$ depends on the same features as $T_{1}$ and also on the concentration of macromolecules in the sample, the pulse sequence and the magnetic environment. The repetition time was $10 \mathrm{~s}$ with a total acquisition time of $2.7 \mathrm{~min}$. It is worth noting that, for the purpose of the present study, a limited 
number of $T_{2}$-weighted images were required to visualise creaming. We have however acquired a longer, complete spin-echo data set, repeated once, should possible future developments (for example, $T_{2}$ mapping) require its evaluation. Volume, $T_{1}$ and $T_{2}$ data sets were acquired at time $t=10,30,50 \mathrm{~min}$ and at $t=70 \mathrm{~min}$ (after drinking the additional water). Volunteers were asked to hold their breath before each image acquisition to minimise diaphragmatic displacement. In between scanning, volunteers were asked to sit upright on the scanning bed, lying down only for the time necessary to acquire the images. We also trained the volunteers to lie on the scanner bed and then to sit up on it without raising the right hand side of the abdomen, as this could have allowed oil layers to float close to the pylorus, which we have previously demonstrated encourages fat emptying (Boulby et al. 1997). This would not happen when normally standing or sitting. Padding placed on the scanner bed helped the subjects to keep the left-hand side of the abdomen higher than the right side when imaged.

\section{Determination of the oil fraction of gastric aspirates}

The EPI measurements in vivo of the oil fraction present in the meal in the gastric lumen (described later) at different time points were compared with bench measurements of the oil fraction of gastric aspirates collected via nasogastric tube at the corresponding time point. Similar measurements were also made on the emulsion meal before ingestion. The gastric aspirates were frozen immediately after collection at $-5^{\circ} \mathrm{C}$ or below and then kept at $-70^{\circ} \mathrm{C}$ or below for later analysis. Prior to bench measurements, the gastric aspirates were brought to room temperature. Each aspirate was gently agitated to obtain homogeneity and the $\mathrm{pH}$ was measured. A sample from each aspirate was taken and the oil droplet size distributions measured. The oil weight fraction $\left(f_{\text {oil }}\right)$ of a $1 \mathrm{ml}$ sample was measured using a standard methanol-chloroform extraction method (Bligh \& Dyer, 1959), and expressed as weight/weight fraction of oil in the whole sample $\left(f_{\text {oil }}=1\right.$ for pure oil and $f_{\text {oil }}=0$ for pure water).

\section{Data acquisition and analysis}

Gastric volume. The good image contrast between the gastric luminal contents and the rest of the body allowed easy and precise measurement of the luminal volume in each slice while excluding organs, gastrointestinal gas and the nasogastric tube. Measurements of the volume of the gastric contents were carried out by manually tracing the region of interest around the meal within the stomach on each image using Analyze software (Biomedical Imaging Resource, Mayo Foundation, Rochester, MN, USA) and summing across all the slices to determine the total meal volume at each time point. For both meals the value of the aqueous phase of the gastric contents for each volunteer was plotted against time and the partial area under the curve calculated between the start and end point determined as:

$$
\begin{gathered}
\text { [Volume }(t=10) \times 40]-[(\text { Volume }(t=10) \\
- \text { Volume }(t=50)) \times 40 / 2]
\end{gathered}
$$

For each meal the mean area under the curve and its standard error, averaged over all six subjects, were calculated.
For comparison of the areas under the curve between different meals, since normal distribution of the data could not be assumed, statistical analysis of related variables was performed using the non-parametric Friedman two-way ANOVA by ranks, followed by the two-tailed Wilcoxon signed rank test for paired comparisons (each volunteer consumed both meals).

Half-life of the meal in the stomach. The half-life of the gastric residence time of the meals $\left(t_{50 \%}\right.$, time to half empty the gastric contents) was estimated by extrapolation from the individual slopes and is presented as mean with its standard error. Significance was calculated by the two-tailed Wilcoxon signed rank test.

Oil volume fraction of gastric contents. $T_{1}$ data sets were processed by tracing a region of interest around the meal within the stomach. The region of interest was drawn omitting the uppermost part of the meal (in order to exclude floating layers). The inversion recovery data collected at different inversion times were then fitted to a simple model as previously described (Marciani et al. 2004). Pearson's product moment correlation was used to assess the significance of agreement between the EPI assessment of oil volume fraction and the oil volume fraction obtained by direct analysis of the gastric aspirates at each time point. Oil volume fraction data are expressed as means with their standard errors.

Gastric aspirate $\mathrm{pH}$ values (means with their standard errors) were compared at each time point using a paired $t$ test assuming equal variances.

\section{Results}

\section{In vitro characterisation of oil-in-water emulsions}

The protocol described allowed the production of two emulsion systems (one acid-stable and one acid-unstable) that had very similar mean oil droplet size $(3.6 \mu \mathrm{m})$ and distribution (Fig. 2). Acidification in vitro of the acid-unstable meal below $\mathrm{pH} 4.0$ resulted in complete destabilisation of the emulsion causing separation of the different phases. By sampling the resulting aqueous phase and analysis by light microscopy it was found that at this low $\mathrm{pH}$ no oil remained dispersed within this phase. The acid-stable meal was acidified to $\mathrm{pH} 2 \cdot 0$, held at this low $\mathrm{pH}$ for $1 \mathrm{~h}$ and then resized. The oil droplet size distribution measured showed no change from the

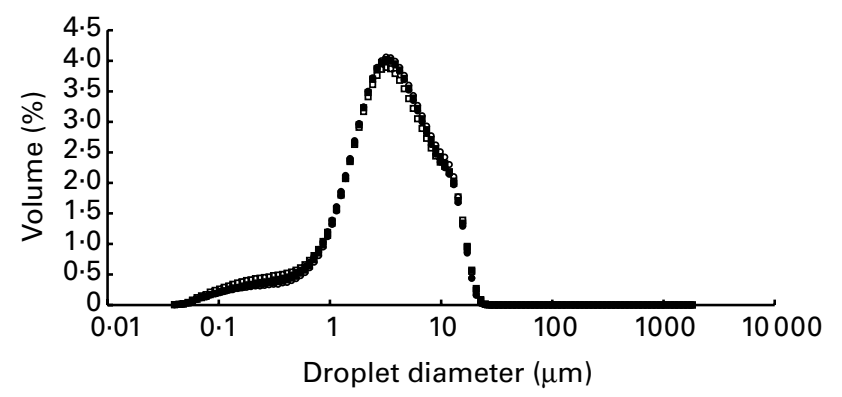

Fig. 2. Oil droplet size distributions of acid-stable emulsion test meals before and after acid treatment. $(\bigcirc)$, After production; $(\bullet)$, after $15 \mathrm{~min}$ incubation;

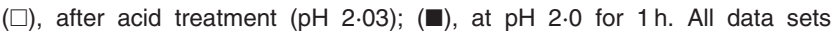
are means of three independent measurements. For details of subjects and procedures, see p. 332 . 
distribution measured prior to acidification (Fig. 2), indicating no separation of oil from the emulsion.

\section{Zeta potential of emulsion droplets}

The influence of $\mathrm{pH}$ on the zeta potential of the emulsion droplets of the acid-stable emulsion was investigated. The zeta potential was measured directly after production of a Tween 60 -stabilised emulsion at native $\mathrm{pH}(5 \cdot 3)$ and then after acidification to $\mathrm{pH} 2.0$ for $1 \mathrm{~h}$. At native $\mathrm{pH}$ the mean zeta potential was -31.85 (SE 1.40$) \mathrm{mV}$ and at $\mathrm{pH} 2.0$ it was -2.5 (SE $0 \cdot 6) \mathrm{mV}$.

\section{In vivo study}

The emulsions were not very palatable because of a bitter taste but this was overcome by the use of coffee flavour and nonenergetic sweetener. All experimental procedures were tolerated well by subjects. There were no adverse events.

\section{In vivo echo-planar imaging}

The oil-in-water emulsion could easily be seen in the gastric lumen and appeared brighter than the surrounding tissues. In the $T_{2}$-weighted images it was possible to observe creaming of the acid-stable emulsion (Fig. 3) and to observe phase separation in the acid-unstable meal. The oil layer in the acidunstable emulsion can be clearly seen in $T_{1}$-weighted images that null the signal from the water phase (Fig. 3(f)).

\section{Gastric emptying}

The average gastric emptying data for the aqueous phase of the gastric contents are shown in Fig. 4. The half-life of the meal in the stomach was estimated by linear extrapolation of the individual aqueous phase volumes (shown as a dashed line in Fig. 4 for the data averaged over all subjects). $t_{50 \%}$ was found to be 107 (SE 11) $\mathrm{min}$ for the acid-stable meal and 84 (SE 14) min for the acid-unstable meal $(P<0.05$, two-tailed Wilcoxon signed rank test). The mean area under the gastric emptying curves for the acid-stable meal was 20464 (SE 1744) $\mathrm{ml} \times$ min and for the acid-unstable meal was 15537 (SE 1966) $\mathrm{ml} \times \min \quad(P>0.028$, two-tailed Wilcoxon signed rank test).

\section{Oil fraction measurements}

In Table 1 the mean oil fractions in the body of the gastric lumen found by EPI are compared with the mean methanol-chloroform extraction measurements of gastric aspirates taken at corresponding times. The correlation between imaging and aspirate data was $r 0.66$ for the acid-stable meal and $r 0.52$ for the acid-unstable meal, showing that EPI can estimate the fraction of oil remaining in the stomach during digestion. Some scatter in the data would have arisen from both aspirate sampling errors and the EPI measurement errors.

\section{pH of aspirates}

In vitro both emulsions gave similar titration curves of $\mathrm{pH}$. volume of $0.1 \mathrm{M}-\mathrm{HCl}$ added, indicating that there was no difference in the buffering capacity of the two systems. The $\mathrm{pH}$ values of the gastric aspirates are shown in Table 2. A paired $t$ test indicates that the $\mathrm{pH}$ values of the gastric aspirates were not significantly different at any time point, although there was a trend for the $\mathrm{pH}$ of the acid-stable emulsion to be consistently lower than that of the acid-unstable emulsion.

\section{Discussion}

Fat enhances the mouth-feel of food (Guinard et al. 1997), which is one reason why a significant proportion of the fat in the modern diet is incorporated within the food structure in the form of surfactant-stabilised lipid emulsions. The emulsifiers that we used in the present study, sorbitan esters and polysorbates (the Spans and Tweens), are used widely to aid stability and add 'gloss' and texture to desserts such as ice cream (Charalambous \& Doxastakis, 1989).

Minor differences in the chemistry of the selected emulsifiers produced two similar emulsions with radically different responses to low $\mathrm{pH}$ in vitro and this behaviour was, as expected, observed in the gastric lumen (Fig. 3(f)). Although in vitro acidification of the acid-unstable emulsion resulted in a quick and total separation of the oil and water phases, and although phase separation is seen clearly in Fig. 3(f), gastric aspirates from the acid-unstable meal in vivo contained some oil. It may be that gastric mixing is inhomogeneous and insufficient to reduce the $\mathrm{pH}$ of the whole gastric contents, leaving some portion of the emulsion unaffected and/or that some of the lipid becomes entrained in gastric mucus and the oil fails to separate as rapidly or cleanly as the in vitro model would suggest. Nevertheless, 10 min after ingestion of the acid-unstable meal the lower aqueous phase contained only about $30 \%$ of its original oil content (Table 1) and this phase emptied from the stomach faster than the acid-stable emulsion. The lower, predominantly aqueous phase may not have contained enough lipid to stimulate a sufficient response from the fatty acid-sensitive, cholecystokinin-containing enteroendocrine cells in the duodenum to mediate a reduction in the rate of gastric emptying (Cote et al. 1995; Santangelo et al. 1998). This would also affect the sense of satiety (Bray, 2000; Mathus-Vliegen et al. 2005). Since the desire to eat is linearly related to the gastric volume (Marciani et al. 2001b) we might predict that the acid-stable meal, because of its slow emptying, will prolong gastric distension and hence also satiety.

In the in vitro characterisation of the acid-stable emulsion, creaming (the concentration of the dispersed oil phase towards the top of the meal) did not occur because the system was gently stirred. However, during in vivo imaging of the acidstable emulsion, creaming within the stomach was observed (Fig. 3) to occur slowly over $50 \mathrm{~min}$, indicating that mixing in the body of the stomach is very gentle and insufficient to prevent this process. Since we collected our gastric aspirates during early gastric emptying $(t=10,30$ and $50 \mathrm{~min})$ some time before $50 \%$ of the acid-unstable meal had emptied $\left(t_{50 \%}=84 \mathrm{~min}\right)$, our samples came from the aqueous phase rather than the floating oil layer of this meal. Therefore, the depletion in lipid content of the gastric aspirates is consistent with creaming and dilution by gastric secretions. An important physical property of colloidal systems is the tendency of the 


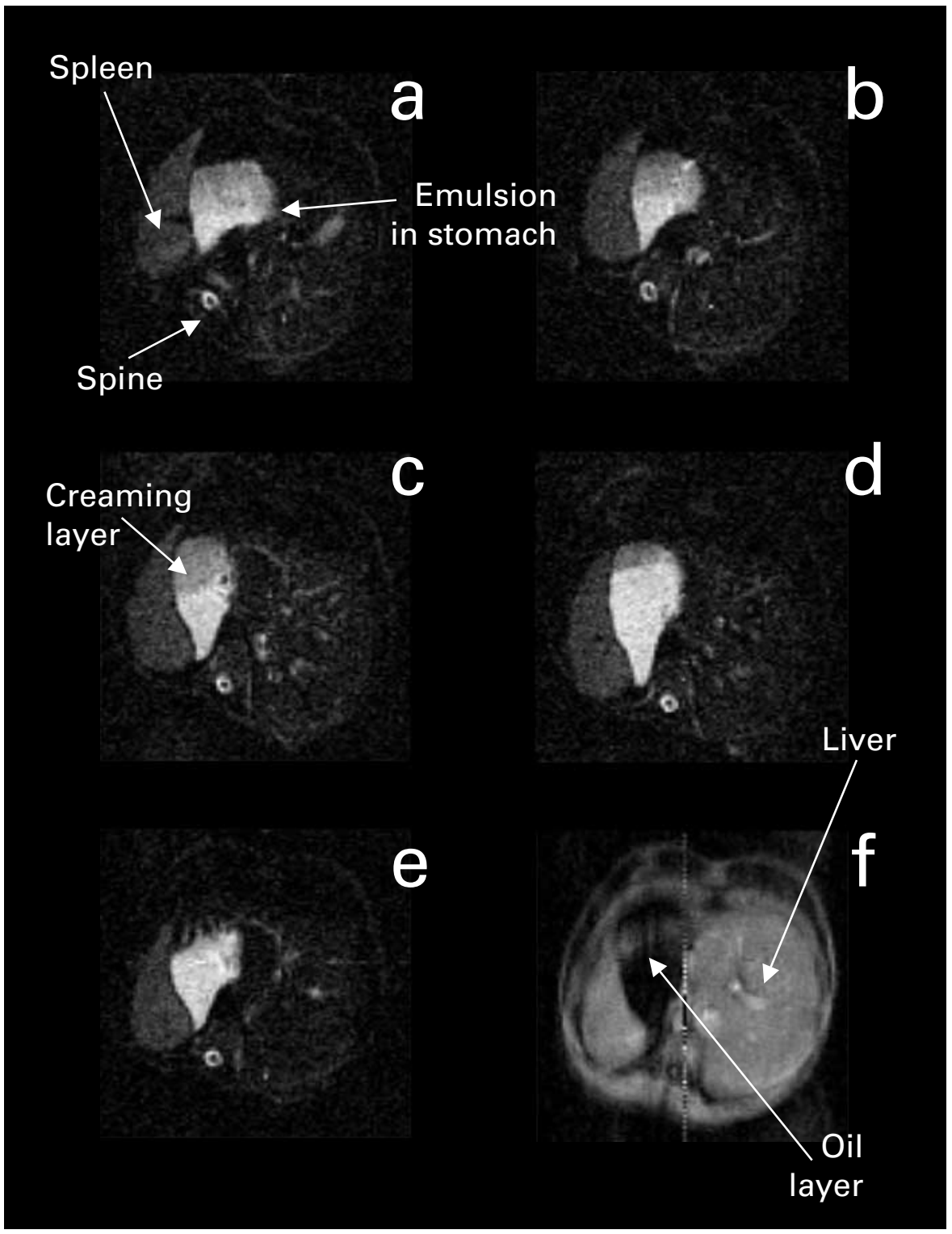

Fig. 3. Example of transverse relaxation time $\left(T_{2}\right)$-weighted spin-echo visualisation, using echo-planar magnetic resonance imaging, of an acid-stable oil emulsion progressively creaming within the gastric lumen of a healthy volunteer with time (t) after ingestion: (a) $t=10 \mathrm{~min}$, (b) $t=30 \mathrm{~min}$, (c) $t=50 \mathrm{~min}$ and (d) $t=70 \mathrm{~min}$. The creaming layer contains more oil droplets and hence appears darker, while the water phase appears brighter. (e) shows an acid-unstable oil emulsion at $t=10 \mathrm{~min}$ after the same volunteer ingested it on a separate occasion. Most of the signal of the oil on top of the water phase is lost due to the delayed acquisition but the oil layer can clearly be seen in a longitudinal relaxation time $\left(T_{1}\right)$-weighted image (f) acquired at the same time with the water phase signal nulled. For details of subjects and procedures, see p. 332

dispersed particles to aggregate as a result of interactions during particle collisions. The aggregates become more buoyant and float to the surface, a process that is described as creaming. Stability of an emulsion system is dominated by repulsive interactions between similarly charged dispersed oil droplets and the zeta potential is a measure of the magnitude of this repulsion. The Tween 60 used in these studies imparts a negative surface charge to the oil droplets at the $\mathrm{pH}$ of the native emulsion, which is how the meals are fed to the volunteers. Upon acidification of the emulsion, as would be expected within the gastric environment, the negative zeta potential (Shaw, 1992) was found to decrease from $-31.85 \mathrm{mV}(\mathrm{pH} 5.2$ ) to $-2.5 \mathrm{mV}$ at $\mathrm{pH} 2.0$. Therefore, as the Tween 60-stabilised emulsion was acidified, the effective surface charge was reduced (by hydrogen ion screening) sufficiently to allow the oil droplets to aggregate (Hunter, 1981) and creaming to occur within the low shear environment of the body of the stomach. However, it would appear that enough surface charge remained on the oil droplets to prevent them from coalescing, even when in close contact with one another.

The oil emulsions used in the present study were model systems, as our main aim was to visualise the in vivo behaviour of different emulsions. This was a pilot study, and hence we chose a small sample $(n$ 6) that we expected to provide sufficient MRI measurements and nasogastric samples to evaluate the technique and provide initial observations on the behaviour of the two emulsion meals in vivo. Preliminary in vitro 


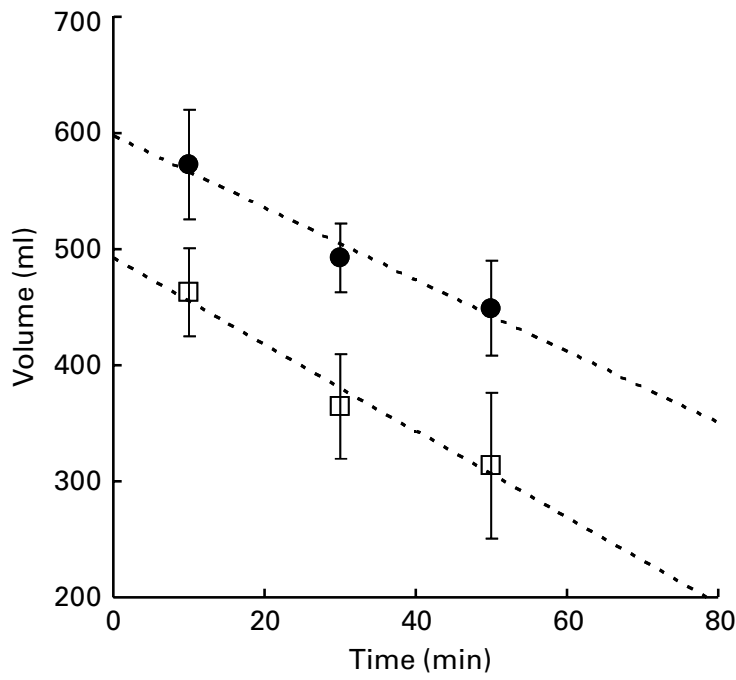

Fig. 4. Average gastric emptying data for the aqueous phase of the gastric contents for the acid-stable $(\bullet)$ and the acid-unstable $(\square)$ emulsion meals. Values are means with their standard error shown by vertical bars; (-.-) show the linear fits to the average data for each meal. For details of subjects and procedures, see p. 332 .

studies suggested that the response of the emulsion to acid is rapid, hence our choice of a short imaging time $(50 \mathrm{~min}$ after meal ingestion). Indeed, significant differences in gastric emptying were detected, with the acid-stable emulsion meal emptying more slowly than the aqueous phase of the acid-unstable meal. However, further studies on larger samples and longer imaging times (lasting at least twice the half-emptying time that was estimated here) are needed to fully investigate the characteristics of gastric emptying. The estimated emptying rate, about $14.7 \mathrm{~kJ}$ per min, appears faster than would be expected and may be due to inaccuracies of the estimation of half emptying since it is based on a short scanning time. A weakness of the study relates to the need for subjects to lie flat while being imaged. This is inevitable given the scanner conformation. However, we trained the volunteers to lie on the scanner bed and then to sit up on it (avoiding also significant movements and walking around the scanner room) without causing oil layers to float into close proximity to the pylorus (Boulby et al. 1997). We also tried to maximise the time the subjects spent upright between scans because this is more physiologically relevant for normal digestion. The sit up/lie down movements and also breathing in the seated position could have introduced some additional mixing of the emulsion meals. However, this additional mixing would have been similar for both experimental days and the subjects acted as their own controls. Increased mixing may accelerate coalescence (shear-induced coalescence) by increasing the number of encounters between droplets, although this was not observed on the bench under continuous stirring. The presence of a nasogastric tube might also have altered gastric emptying although others have suggested this effect is quite small (Fone et al. 1991) and the effect would have been the same for the two meals; hence the tube should have introduced no bias.

The good correlation between the imaging and the bench measurements of the oil fraction present in the emulsion within the gastric lumen indicated that EPI can be used to monitor non-invasively the behaviour of emulsions in the stomach. Some data scatter in the oil fractions measured before ingestion was expected since this may arise both from errors in sampling the emulsion for the bench measurements and from errors in the EPI measurements.

Although robust and accurate, the inversion recovery EPI method we used required minutes to collect data on a single imaging plane. The use of faster techniques may allow multi-slice investigation of spatial variations of oil fraction and meal mixing in different regions of the stomach. This could be achieved using Look-Locker EPI (Freeman et al. 1998) or inversion recovery TrueFISP (Scheffler \& Hennig, 2001) methods.

As diseases of overnutrition are becoming increasing prevalent, it is necessary to study carefully how different food preparation methods affect satiety and the postprandial delivery of fats to the intestine. The present study suggests that the emulsifier could be used to manipulate gastric emptying - and

Table 1. Comparison between in vivo measurements of the fraction of oil in the emulsions in the gastric lumen at different times by echo-planar magnetic resonance imaging (EPI) and bench measurements on corresponding nasogastric aspirates

(Mean values and their standard errors for six subjects)

\begin{tabular}{|c|c|c|c|c|c|c|c|c|}
\hline & \multicolumn{4}{|c|}{ Acid-stable emulsion } & \multicolumn{4}{|c|}{ Acid-unstable emulsion } \\
\hline & \multicolumn{4}{|c|}{ Oil fraction* measured by } & \multicolumn{4}{|c|}{ Oil fraction* measured by } \\
\hline & \multicolumn{2}{|c|}{ EPI† } & \multicolumn{2}{|c|}{ Aspirates } & \multicolumn{2}{|c|}{ EPI } & \multicolumn{2}{|c|}{ Aspirates } \\
\hline & Mean & SE & Mean & SE & Mean & SE & Mean & SE \\
\hline Before ingestion & 0.116 & 0.008 & $0 \cdot 140$ & 0.003 & $0 \cdot 15$ & 0.03 & 0.133 & 0.006 \\
\hline \multicolumn{9}{|c|}{ Time after ingestion (min) } \\
\hline 10 & 0.11 & 0.01 & 0.09 & 0.02 & 0.04 & 0.01 & 0.05 & 0.01 \\
\hline 30 & 0.08 & 0.01 & 0.08 & 0.01 & 0.04 & 0.03 & 0.04 & 0.03 \\
\hline 50 & 0.07 & 0.01 & 0.09 & 0.02 & 0.02 & 0.01 & 0.03 & 0.02 \\
\hline 70 & 0.06 & 0.01 & 0.042 & 0.007 & 0.012 & 0.007 & 0.05 & 0.04 \\
\hline
\end{tabular}

${ }^{*}$ Weight/weight fraction of oil in the samples defined as $f=0$ for pure water and $f=1$ for pure oil.

† Correlation between the values measured in vivo in the gastric lumen by EPI and by methanol-chloroform extraction of the corresponding nasogastric aspirates is Pearson's $r 0.66$.

$\ddagger$ Correlation between the values measured in vivo in the gastric lumen by EPI and by methanol-chloroform extraction of the corresponding nasogastric aspirates is Pearson's $r 0.52$.

For details of subjects and procedures, see p. 332. 
Table 2. $\mathrm{pH}$ of the nasogastric aspirates (Mean values and their standard errors for six subjects)

\begin{tabular}{|c|c|c|c|c|}
\hline & \multicolumn{2}{|c|}{$\begin{array}{l}\text { Acid-stable } \\
\text { emulsion }\end{array}$} & \multicolumn{2}{|c|}{$\begin{array}{l}\text { Acid-unstable } \\
\text { emulsion }\end{array}$} \\
\hline & Mean & SE & Mean & SE \\
\hline Before ingestion & $5 \cdot 39$ & 0.06 & $5 \cdot 21$ & 0.09 \\
\hline \multicolumn{5}{|c|}{ Time after ingestion (min) } \\
\hline 10 & 3.9 & 0.6 & $5 \cdot 2$ & 0.8 \\
\hline 30 & $2 \cdot 6$ & 0.2 & $3 \cdot 1$ & 0.6 \\
\hline 50 & $2 \cdot 6$ & 0.3 & $3 \cdot 8$ & 0.9 \\
\hline 70 & 3.5 & 0.7 & $5 \cdot 6$ & 0.8 \\
\hline
\end{tabular}

For details of subjects and procedures, see p. 332 .

hence, in turn, the desire to eat - in the postprandial period, enabling the production of foods that are both palatable and maximise and prolong satiety. The present study also suggests that when performing feeding studies aimed at measuring the 'bioavailability' of a dietary compound by plasma appearance, it is not sufficient to simply assume a uniform rate of delivery over the period of gastric emptying but it is necessary to consider whether redistribution or phase separation has occurred in the gastric lumen. A similar observation was reported recently for a solid meal (Kunz et al. 2005).

Our study shows that it is possible to use the acid stability of emulsifiers to control the behaviour of oil emulsions in vivo and to monitor in vivo, non-invasively, the intragastric behaviour of oil-in-water emulsions of different gastric acid stability using MRI.

\section{Acknowledgements}

The authors thank Macphie, Glenbervie, Scotland for their kind gift of Tween 60 emulsifier. This research was supported by the Biotechnology and Biological Sciences Research Council (Swindon, UK), Research Grant D14043. The authors thank the volunteers for their participation in this study.

\section{References}

Bligh EG \& Dyer WJ (1959) A rapid method of total lipid extraction and purification. Can J Biochem Biophys 37, 911-917.

Borovicka J, Schwizer W, Guttmann G, Hartmann D, Kosinski M, Wastiel C, Bischof-Delaloye A \& Fried M (2000) Role of lipase in the regulation of postprandial gastric acid secretion and emptying of fat in humans: a study with orlistat, a highly specific lipase inhibitor. Gut 46, 774-781.

Boulby P, Gowland P, Adams V \& Spiller RC (1997) Use of echo planar imaging to demonstrate the effect of posture on the intragastric distribution and emptying of an oil/water meal. Neurogastroenterol Motil 9, 41-47.

Boulby P, Moore R, Gowland P \& Spiller RC (1999) Fat delays emptying but increases forward and backward antral flow as assessed by flow-sensitive magnetic resonance imaging. Neurogastroenterol Motil 11, 27-36.

Bray GA (2000) Afferent signals regulating food intake. Proc Nutr Soc 59, 373-384.

Carney BI, Jones KL, Horowitz M, Sun WM, Penagini R \& Meyer JH (1995) Gastric emptying of oil and aqueous meal components in pancreatic insufficiency: effects of posture and on appetite. Am J Physiol 268, G925-G932.
Charalambous G \& Doxastakis G (1989) Food Emulsifiers: Chemistry, Technology, Functional Properties and Applications. Oxford: Elsevier.

Christian PE, Moore JG \& Datz FL (1984) Comparison of Tc-99m labeled liver and liver pate as markers for solid-phase gastric-emptying. J Nucl Med 25, 364-366.

Collins PJ, Horowitz M \& Chatterton BE (1988) Proximal, distal and total stomach emptying of a digestible solid meal in normal subjects. Br J Radiol 61, 12-18.

Cote F, Pare P \& Friede J (1995) Physiological effect of cholecystokinin on gastric-emptying of liquid in functional dyspepsia. Am J Gastroenterol 90, 2006-2009.

Cunningham KM, Baker RJ, Horowitz M, Maddox AF, Edelbroek MAL \& Chatterton BE (1991) Use of technetium-99m(V)thiocyanate to measure gastric-emptying of fat. $J$ Nucl Med 32, 878-881.

Department for Environment Food and Rural Affairs (2000) SN 4512 - National Food Survey 2000. http://www.data-archive.ac.uk

Dickinson E (2003) Hydrocolloids at interfaces and the influence on the properties of dispersed systems. Food Hydrocolloids 17, $25-39$.

Drewnowski A (1997) Why do we like fat?" J Am Diet Assoc 97, Suppl., S58-S62.

Edelbroek M, Horowitz M, Maddox A \& Bellen J (1992) Gastric emptying and intragastric distribution of oil in the presence of a liquid or a solid meal. J Nucl Med 33, 1283-1290.

Evans DF, Lamont G, Stehling MK, Blamire AM, Gibbs P, Coxon R, Hardcastle JD \& Mansfield P (1993) Prolonged monitoring of the upper gastrointestinal tract using echo-planar magnetic resonance imaging. Gut 34, 848-852.

Fone DR, Horowitz M, Heddle R, Maddox AF, Collins PJ, Read NW \& Dent J (1991) Comparative effects of duodenal and ileal intubation on gastric emptying and postprandial antral, pyloric, and duodenal motility. Scand J Gastroenterol 26, 16-22.

Fraser R, Borovicka J, Schwizer W, Asal K, Crelier G, Boesiger P, Blum AL, Gonvers JJ \& Fried M (1993) Magnetic resonance imaging (MRI) to measure proximal and distal gastric motility during emptying of a liquid meal. Gastroenterology 104, A509-A509.

Freeman AJ, Gowland PA \& Mansfield P (1998) Optimization of the ultrafast Look-Locker echo-planar imaging T1 mapping sequence. Magn Reson Imaging 16, 765-772.

Guinard JX, ZoumasMorse C, Mori L, Uatoni B, Panyam D \& Kilara A (1997) Sugar and fat effects on sensory properties of ice cream. $J$ Food Sci 62, 1087-1094.

Heading RC, Tothill P, Laidlow AJ \& Shearman DJC (1971) An evaluation of 113-In-DTPA chelate in the measurement of gastric emptying by scintiscanning. Gut 12, 611-615.

Horowitz M, Collins PJ \& Shearman DJC (1985) Disorders of gastric emptying in humans and the use of radionuclide techniques. Arch Intern Med 145, 1467-1472.

Horowitz M, Jones K, Edelbroek MAL, Smout A \& Read NW (1993) The effect of posture on gastric-emptying and intragastric distribution of oil and aqueous meal components and appetite. Gastroenterology 105, 382-390.

Howseman AM, Stehling MK, Chapman B, Coxon R, Turner R, Ordidge RJ, Cawley MG, Glover P, Mansfield P \& Coupland RE (1988) Improvements in snap-shot nuclear magnetic resonance imaging. Br J Radiol 61, 822-828.

Hunter RJ (1981) Zeta Potential in Colloid Science, Principles and Applications. London: Academic Press.

Jian R, Vigneron N, Najean Y \& Bernier JJ (1982) Gastric-emptying and intra-gastric distribution of lipids in man: a new scintigraphic method of study. Dig Dis Sci 27, 705-711.

Kerlin P, Byrnes O \& Wong L (1989) Radiolabeled unprocessed bran: validation of a practical labeling technique and evaluation of gastrointestinal transit profiles in health. J Gastroenterol Hepatol 4, 119-126. 
Kunz P, Feinle-Bisset C, Faas H, Boesiger P, Fried M, Steingotter A \& Schwizer W (2005) Effect of ingestion order of the fat component of a solid meal on intragastric fat distribution and gastric emptying assessed by MRI. J Magn Reson Imaging 21, 383-390.

Lin HC, Doty JE, Reedy TJ \& Meyer JH (1990) Inhibition of gastricemptying by sodium oleate depends on length of intestine exposed to nutrient. Am J Physiol 259, G1031-G1036.

Lissner L \& Heitmann BL (1995) Dietary fat and obesity: evidence from epidemiology. Eur J Clin Nutr 49, 79-90.

Malmud LS, Fisher RS, Knight LC \& Rock E (1982) Scintigraphic evaluation of gastric emptying. Semin Nucl Med 12, 116-125.

Marciani L, Gowland PA, Spiller RC, Manoj P, Moore RJ, Young P \& Fillery-Travis AJ (2001b) Effect of meal viscosity and nutrients on satiety, intragastric dilution, and emptying assessed by MRI. Am J Physiol 280, G1227-G1233.

Marciani L, Wickham M, Hills BP, Wright J, Bush D, Faulks R, FilleryTravis A, Spiller RC \& Gowland PA (2004) Intragastric oil-in-water emulsion fat fraction measured using inversion recovery echo-planar magnetic resonance imaging. J Food Sci 69, E290-E296.

Marciani L, Young P, Wright J, Moore R, Coleman N, Gowland PA \& Spiller RC (2001a) Antral motility measurements by magnetic resonance imaging. Neurogastroenterol Motil 13, 511-518.

Mathus-Vliegen EMH, Van Ierland-Van Leeuwen ML \& Roolker W (2005) Gastric emptying, CCK release, and satiety in weight-stable obese subjects. Dig Dis Sci 50, 7-14.
Meyer JH, MacGregor IL, Gueller R, Martin P \& Cavalieri R (1976) Tc-99-m tagged chicken liver as a marker of solid food in the human stomach. Dig Dis Sci 21, 296-304.

Mokdad AH, Ford ES, Bowman BA, Dietz WH, Vinicor F, Bales VS \& Marks JS (2003) Prevalence of obesity, diabetes, and obesityrelated health risk factors, 2001. J Am Med Assoc 289, 76-79.

Pilichiewicz A, O'Donovan D, Feinle C, Lei Y, Wishart JM, Bryant L, Meyer JH, Horowitz M \& Jones KL (2003) Effect of lipase inhibition on gastric emptying of, and the glycemic and incretin responses to, an oil/aqueous drink in type 2 diabetes mellitus. $J$ Clin Endocrinol Metab 88, 3829-3834.

Santangelo A, Peracchi M, Conte D, Fraquelli M \& Porrini M (1998) Physical state of meal affects gastric emptying, cholecystokinin release and satiety. Br J Nutr $\mathbf{8 0}, 521-527$.

Scheffler K \& Hennig J (2001) $T_{1}$ quantification with inversion recovery TrueFISP. Magn Reson Med 45, 720-723.

Schwizer W, Maecke H \& Fried M (1992) Measurement of gastric emptying by magnetic resonance imaging in humans. Gastroenterology 103, 369-376.

Shaw DJ (1992) Colloid and Surface Chemistry, pp. 174-209. Oxford: Butterworth-Heinemann Ltd.

Stehling MK, Evans DF, Lamont G, Ordidge RJ, Howseman AM, Chapman B, Coxon R, Mansfield P, Hardcastle JD \& Coupland RE (1989) Gastrointestinal tract: dynamic MR studies with echoplanar imaging. Radiology 171, 41-46. 